\title{
Sustainable, bio-based silicone with layered double hydroxide hybrid and their application in natural-fiber reinforced phenolic composites with enhanced performance
}

Cheng $\mathrm{Li}^{a}$, Jintao Wan ${ }^{b}$, Ye-Tang Pan ${ }^{b}$, Peng-Cheng Zhao ${ }^{c}$, Hong Fan ${ }^{a}{ }^{*}$, De-Yi Wang $^{b, *}$

a State Key Laboratory of Chemical Engineering, College of Chemical and Biological Engineering, Zhejiang University, Zheda Road 38 \#, Hangzhou 310027, China

b IMDEA Materials Institute, C/ Eric Kandel, 2, Getafe, Madrid 28906, Spain

c Leibnitz Institute of Polymer Research Dresden, Hohe Strasse 6, Dresden, D-01069, Germany

*Corresponding Authors. E-mail: hfan@zju.edu.cn; deyi.wang@imdea.org.

Total number of pages: 4

Total number of Figures: 2

Total number of Tables: 1 . 


\section{XRD characterization of LDH}

Sample preparation: The LDHs were mixed with phenolic resin (PF/SPF) by solvent mixing (weight ratio: $\mathrm{LDH}: \mathrm{PF}=1: 9$, with acetone as solvent), and the solvent was removed by rotovap. Then the sample were pulverized, and mixed with HMTA (weight ratio: $\mathrm{PF}: \mathrm{HMTA}=9: 1$ ), to run $\mathrm{XRD}$ tests. The curing process of $\mathrm{LDHs} / \mathrm{PF}$ samples were conducted in $180{ }^{\circ} \mathrm{C}$ for three hours.

According to the WAXS spectra, the LDH layer distance is neither affected by phenolic resin type (PF or SPF) nor the curing process (Fig. S1, Fig. S2 and Tab. S1). The layer distance of NLDH in PF-NLDH are $0.89 \mathrm{~nm}$ (before curing) and $0.91 \mathrm{~nm}$ (after curing), and the first basal reflection peaks (003) appear at $9.92^{\circ}$ (20 angle, before curing) and $9.76^{\circ}$ (after curing), respectively. In SPF, the layer distance of $\mathrm{NLDH}$ are $0.88 \mathrm{~nm}$ (before curing) and $0.89 \mathrm{~nm}$ (after curing), and the first basal reflection peaks (003) appear at $9.95^{\circ}$ (20 angle, before curing) and $9.88^{\circ}$ (after curing), respectively. The layer distance of SDBSLDH in PF-SDBSLDH are $2.99 \mathrm{~nm}$ (before curing) and $3.07 \mathrm{~nm}$ (after curing), and the first basal reflection peaks (003) appear at $2.96^{\circ}$ ( $2 \theta$ angle, before curing) and $2.88^{\circ}$ (after curing), respectively. In $\mathrm{SPF}$, the layer distance of SDBSLDH are $3.01 \mathrm{~nm}$ (before curing) and $3.03 \mathrm{~nm}$ (after curing), and the first basal reflection peaks (003) appear at $9.04^{\circ}$ (20 angle, before curing) and $8.87^{\circ}$ (after curing), respectively. 


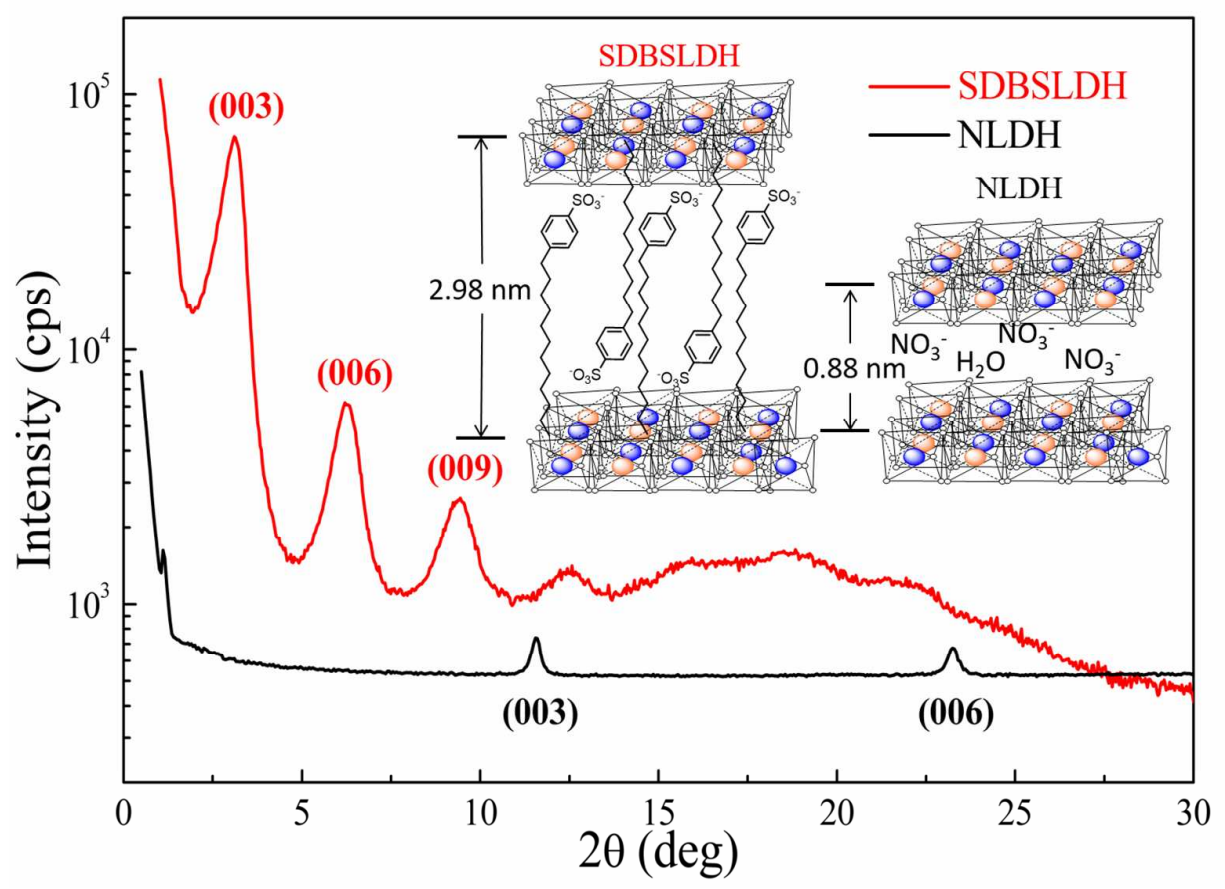

Figure S1. WAXS graphs for LDHs

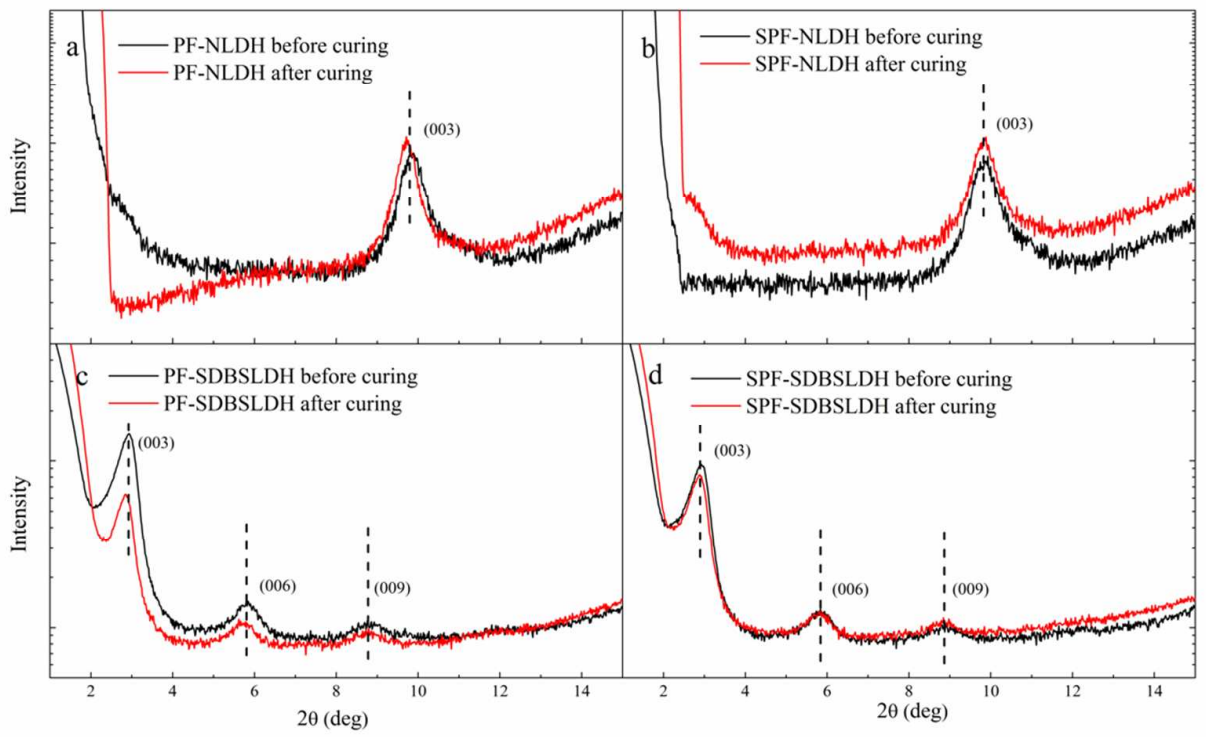

Figure S2. WAXS graphs for LDH contained phenolic resins 
Table S1. WAXS data for LDHs and LDH contained phenolic resins

\begin{tabular}{|c|c|c|c|c|}
\hline Samples & $\begin{array}{l}\text { First basal } \\
\text { reflection peaks } \\
\text { before curing }\left({ }^{\circ}\right)\end{array}$ & $\begin{array}{l}\text { First basal } \\
\text { reflection } \\
\text { after curing }\left({ }^{\circ}\right)\end{array}$ & $\begin{array}{l}\text { Layer distance of } \\
\text { LDH before } \\
\text { curing }(\mathrm{nm})\end{array}$ & $\begin{array}{l}\text { Layer distance of } \\
\text { LDH after curing } \\
(\mathrm{nm})\end{array}$ \\
\hline NLDH & $10.01 \pm 0.08$ & & $0.88 \pm 0.02$ & \\
\hline PF-NLDH & $9.92 \pm 0.06$ & $9.76 \pm 0.06$ & $0.89 \pm 0.01$ & $0.91 \pm 0.01$ \\
\hline SPF-NLDH & $9.95 \pm 0.06$ & $9.88 \pm 0.06$ & $0.88 \pm 0.01$ & $0.89 \pm 0.01$ \\
\hline SDBSLDH & $2.98 \pm 0.02$ & & $2.98 \pm 0.02$ & \\
\hline PF-SDBSLDH & $2.96 \pm 0.03$ & $2.88 \pm 0.03$ & $2.99 \pm 0.02$ & $3.07 \pm 0.03$ \\
\hline SPF-SDBSLDH & $2.94 \pm 0.02$ & $2.92 \pm 0.02$ & $3.01 \pm 0.02$ & $3.03 \pm 0.03$ \\
\hline
\end{tabular}

